\title{
PERFORMANCE ENHANCEMENT USING SELECTIVE REINFORCEMENT FOR METALLIC SINGLE- AND MULTI-PIN LOADED HOLES
}

\author{
Gary L. Farley* \\ Army Research Laboratory \\ Vehicle Technology Directorate \\ Langley Research Center \\ Hampton, VA 23681 \\ and \\ Banavara R. Seshadri ${ }^{\dagger}$ \\ National Institute of Aerospace \\ Langley Research Center \\ Hampton, VA 23681
}

\begin{abstract}
An analysis based investigation of aluminum with metal matrix composite selectively reinforced single- and multi-hole specimens was performed and their results compared with results from geometrically comparable non-reinforced specimens. All reinforced specimens exhibited a significant increase in performance. Performance increase of up to 170 percent was achieved. Specimen failure modes were consistent with results from reinforced polymeric matrix composite specimens. Localized reinforcement application (circular) proved as effective as a broader area (strip) reinforcement. Also, selective reinforcement is an excellent method of increasing the performance of multi-hole specimens.
\end{abstract}

\section{Introduction}

Metallic materials for aerospace application, in particular aluminum, have experienced increasing competition from polymeric matrix composite (PMC) materials. The most recent gain by the PMC industry is the extensive application of PMC materials on the Airbus A380 and the Boeing 787 aircraft. These gains by the PMC industry has resulted in a loss of market share by the aerospace aluminum industry.

Alcoa, a leading international supplier of aluminum to the aerospace industry, has developed a strategy to counter this competition focusing around a triad of technologies, new alloys (Al-Li), integrally stiffened structures and selective reinforcement ${ }^{1}$. The concept of selective reinforcement (SR) is the local application of reinforcement to a base structure (can be a conventional design) to compensate for deficiencies, such as strength, stiffness, durability or damage tolerance ${ }^{1-3}$. Selective reinforcement is a weight-efficient technique for enhancing the structural performance of conventional metallic structures ${ }^{1-3}$. Two selective reinforcement approaches are being pursued; one is by lamination of reinforcement material, such as fiber metal laminate, to a conventional base metal structure $^{1}$. The second approach is by volumetric replacement of the base material with a reinforcement ${ }^{2,3}$ referred to as in situ selective reinforcement. In situ selective reinforcing material could be another metal, a fiber or a particulate.

Alcoa's lamination SR approach in concert with new alloys and integral stiffening has resulted in exceeding their initial 20 percent weight and 20 percent fabrication cost saving goals. These goals sought parody with the performance of PMC materials. In situ selective reinforcement, a less mature approach, offers potentially higher

\footnotetext{
${ }^{*}$ Aerospace Engineer, Mechanics of Structures and Materials Branch, Non-AIAA member

${ }^{\dagger}$ Aerospace Engineer, Mechanics of Structures and Materials Branch, Non-AIAA member
} 
performance gains than lamination. For instance, in situ selective reinforced aluminum panels with cutouts has been shown to exhibit specific buckling and post-buckling response of 20 to over 80 percent higher than the performance of non-reinforced aluminum panels ${ }^{2}$. These SR metal panels even exhibited superior specific performance relative to quasi-isotropic carbon fiber reinforced PMC panels of comparable geometry ${ }^{2}$.

Selective reinforcement has also been shown to be an effective method to slow and even arrest fatigue crack growth in metallic materials ${ }^{1,3}$. In-situ selective reinforcement has also demonstrated a 20 percent improvement in fracture strength and over a 100 percent increase in critical crack length ${ }^{3}$. Load sharing between the reinforcement and the base metallic material is the mechanism associated with improvement in fatigue and fracture response.

Geometric discontinuities and the associated load path shift can be significant structural design challenges. For instance, fastener holes are common geometric discontinuities and potential failure sites on an aircraft. To compensate for the high localized stresses and potential damage initiation that can occur at holes, the thickness of the structure may need to be increased resulting in higher structural weight. Padawer sought to improve bolted joint strength of carbon fiber reinforced PMC material by embedding in the PMC laminate a thin boron-coated Kapton film reinforcing strip in the region of the holes ${ }^{4}$. Reinforcement represented between 6 and 12 percent of the total material in the joint. Fastener-hole strength was increased in excess of 50 percent and strength to weight index twice that of non-reinforced specimens was obtained. It is not unrealistic to believe that reinforcement of metallic structure with holes would benefit from selective reinforcement. However, further understanding of the influence of reinforcement architecture on response and failure mechanisms is required before SR can be commercially applied at fastener holes.

This paper describes the results of an analysis based investigation of the material and structural response of insitu selectively reinforced pin-loaded holes. Single- and multi-pin loaded holes are analyzed with and without selective reinforcement. Reinforcement was 50 percent volume fraction alumina fiber in an 1100 alloy aluminum matrix. The effect of reinforcement thickness, as a percent of the total thickness, as well as reinforcement geometries is investigated. Load-displacement response, maximum load and failure modes are predicted for these pin-loaded holes using a progressive failure analysis integrated into a commercial finite element computer code.

\section{Scope of investigation}

The scope of this investigation focuses on determining the effects of in-situ SR on maximum load carrying capability and determining the failure modes exhibited by the SR pin-loaded hole specimens. These results are compared to the response exhibited by geometrically comparable non-reinforced aluminum specimens.

In-situ SR is modeled as a volumetric replacement of the base aluminum on the surface of the specimen, such as depicted in Fig. 1, forming a constant thickness specimen. Two different reinforcement total thicknesses (20 and 40 percent of the thickness of the specimen) were included in this investigation. Individual reinforcement layer thicknesses for 4 reinforcement layers in a specimen were 5 and 10 percent, respectively, of the total specimen thickness.

Two reinforcement architectures were investigated and are depicted in Fig. 1. The first architecture is a rectangular strip that extended laterally across the specimen, edge to edge. The second reinforcement architecture investigated is a circular ring of reinforcement three times the diameter of the fastener. The circular reinforcement constitutes less than $1 / 3^{\text {rd }}$ of the reinforcement area of the strip reinforcement.

Two specimen related geometrical parameters included in this investigation are specimen width (W) to hole diameter (d), denoted as W/d, and distance from hole center to edge of specimen (e) to hole diameter, denoted as (e/d). The W/d and e/d values used are 2, 4 and 10 and 1,3,4, and 5, respectively. Typical finite element mesh for single-hole specimens are presented in Fig. 2.

Many applications of joints require multiple fasteners in a row and even multiple rows of fasteners. The proximity of multiple holes can cause an adverse interaction of stress states resulting in reduced load carrying capability per fastener. Therefore, in addition to the case of a single hole in a specimen, a series of specimens with multiple holes representative of a row of fasteners are evaluated. This investigation exclusively uses circular reinforcements applied at each hole in the multi-hole specimen. The cases evaluated are; holes in-line, row of holes 
(one row), rows-line of holes (two rows and two holes in a line) and staggered holes (two rows). Finite element mesh for multi-hole specimens is also depicted in Fig. 2.

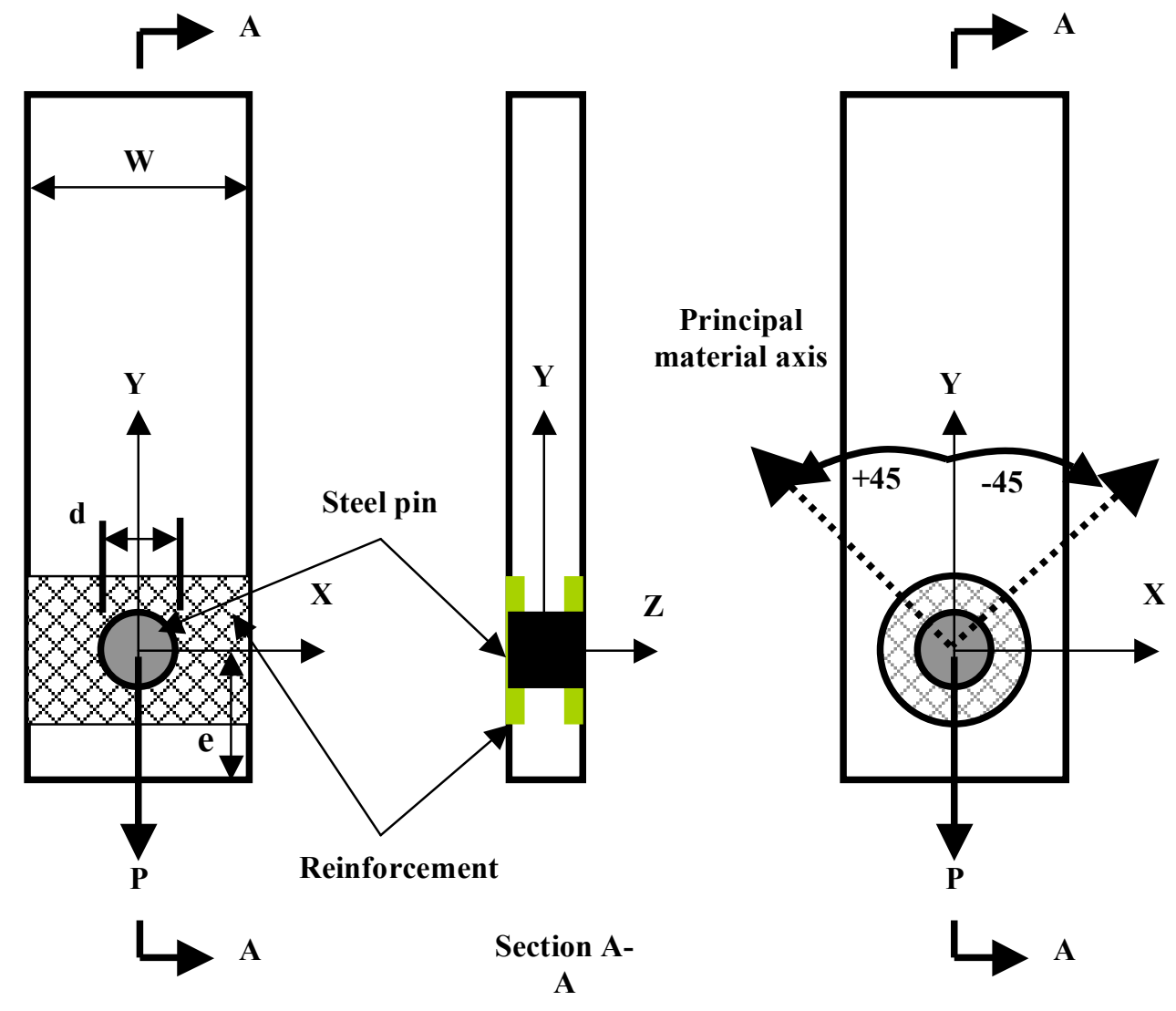

Strip reinforced hole

Circular reinforced hole region region

Figure 1. Typical specimen configuration of single-hole specimens. 


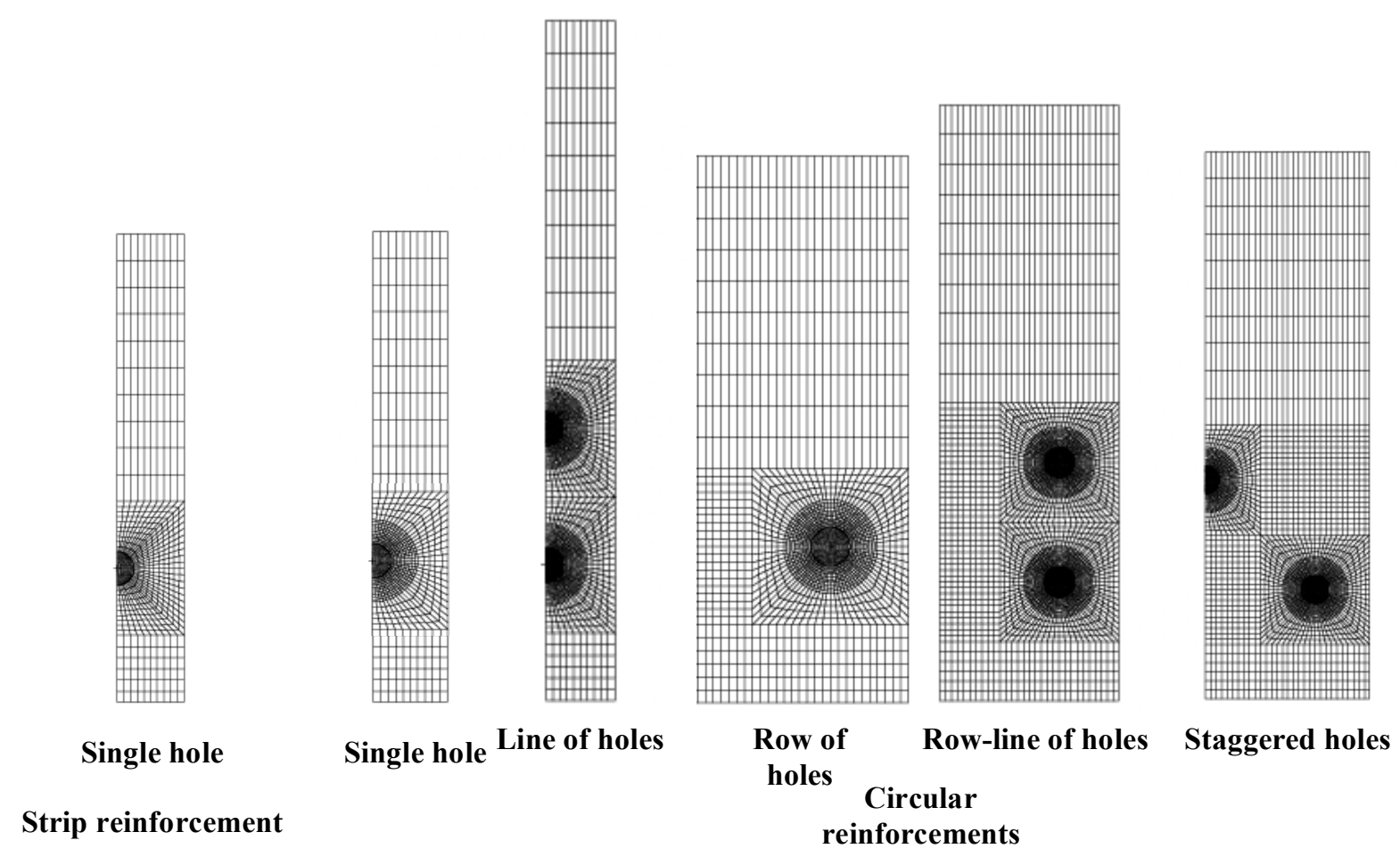

Figure 2. Finite element mesh of single- and multi-hole specimens.

\section{Finite element models}

The specimen is modeled as a reinforced rectangular plate with a circular hole filled with a rigid pin. Four node constant stress plate finite elements (S4 finite elements in ABAQUS ${ }^{5}$ ) are used in all finite element models. No out of plane displacements are allowed. Geometric symmetry (Y-Z plane and X-Y plane) is exploited wherever possible to reduce the computational size of the model. The reinforced region is modeled as a three-layer laminate (symmetric through the thickness) consisting of two surface reinforcement layers, oriented at \pm 45 degrees relative to the longitudinal axis of the specimen with a non-reinforced aluminum layer beneath the reinforcement. The nonreinforced aluminum beneath the reinforcement is thinner than the aluminum outside of the reinforced region to accommodate the in situ reinforcement, as depicted in Fig. 1.

No clearance exists between the pin and the hole. Pin-hole interaction is modeled as a contact problem to accurately represent the local load transfer and predict the stress state near the hole. The rigid pin is constrained from movement while a displacement is applied at the opposite end of the specimen.

The SR layers are representative of a unidirectional reinforcement consisting of alumina ceramic fiber infiltrated with an 1100 alloy aluminum matrix having a fiber volume fraction of 50 percent. Material mechanical properties used for the SR material are, $\mathrm{E}_{11}=35 \mathrm{Msi}, \mathrm{E}_{22}=19 \mathrm{Msi}, \mathrm{G}_{12}=7.4 \mathrm{Msi}, v_{12}=0.275, \varepsilon_{11}=0.0071 \mathrm{in} / \mathrm{in}, \varepsilon_{22}=0.0011 \mathrm{in} / \mathrm{in}$ and $\gamma_{12}=0.0027 \mathrm{in} / \mathrm{in}$. The symbols $\mathrm{E}_{11}$ and $\mathrm{E}_{22}$ are the Young's modulus parallel and perpendicular, respectively, to the fibers, $G_{12}$ is the in plane shear modulus, $n_{12}$ is the in-plane Poisson's ratio and $\varepsilon_{11}, \varepsilon_{22}$ and $\gamma_{12}$ are the failure strains parallel to the fiber, perpendicular to the fiber and in plane shear, respectively. Material properties for the SR material were assumed to be linear elastic to failure. The non-reinforced portion of the SR specimens and the nonreinforced specimens are modeled as composed of 7075-T6 alloy aluminum. The material properties used for the 7075-T6 Al are E=10 Msi, $v=0.3$ where E and $v$ are the Young's modulus and Poisson's ratio, respectively. An elastic-plastic response is assumed with the yield stress of $70 \mathrm{Ksi}$ and a failure strain of $\varepsilon=0.025 \mathrm{in} / \mathrm{in}$. 


\section{Analysis method}

Three distinct structural failure modes; net-tension, shear-out and bearing, are typically exhibited by pin-loaded hole specimens in fiber reinforced composite materials as depicted in Fig. 3. It is reasonable to expect the occurrence of these modes as well as local yielding in the pin-loaded aluminum specimens. Predicting these modes require the ability to predict the elastic and inelastic response as well as progressive material failure of the different materials in the specimen.

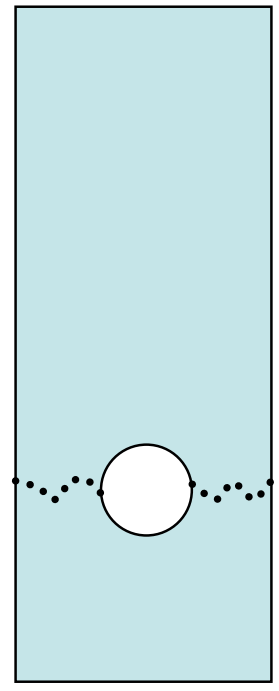

Net-tension

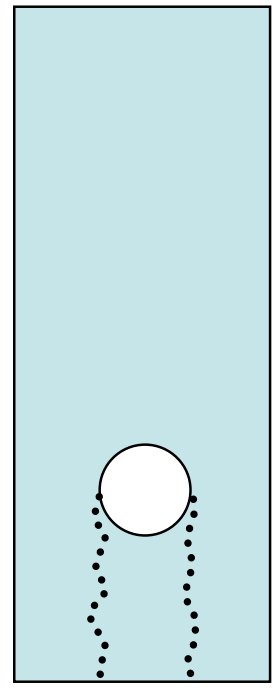

Shear-out

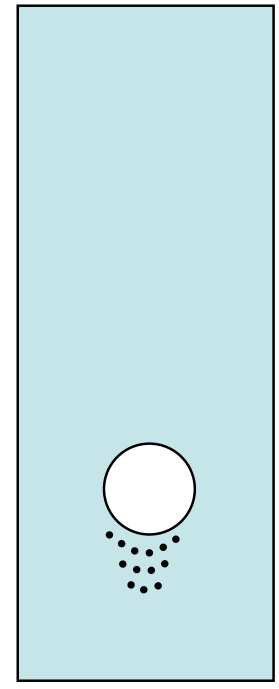

Bearing

Figure 3. Three typical structural failure modes exhibited by pin-loaded hole specimens.

A progressive failure analysis is integrated in the ABAQUS finite element computer program to predict this complex structural response. No pre-defined failure path or region is a priori defined in the model. Material nonlinear properties are incorporated in the analysis to predict the elastic and inelastic response. The Hashin polynomial-based failure criterion is used to predict material failure stress and material failure mode. This failure criterion accurately captures all of the material failure mechanisms associated with pin-loaded hole specimens ${ }^{6}$. Dependent upon the material failure mode, selected material stiffness properties are set to zero in the failure analysis routine. For instance, when a matrix failure occurs transverse to the reinforcement material's principal material axis, in tension or compression, the reinforcement material extensional stiffness transverse to the principal material axis $\left(E_{22}\right)$ set to zero. This material mode is referred to as a transverse failure. A material fiber-matrix shear failure results in shear modulus $\left(\mathrm{G}_{12}\right)$ set to zero and referred to as a shear failure. A fiber compression (bearing) failure results in setting the extension modulus $\mathrm{E}_{11}$ set to zero and referred to as a compression failure. For any failure mode the Poisson's ratio is set to zero.

The analysis procedure consists of incremental application of displacement at the non-pin end of the specimen keeping the pin restrained. Material properties are updated based upon the stress-strain state and failure analysis of each element. After the material properties are updated, the analysis iterates to a converged solution before the next increment of displacement is applied. Reaction forces are calculated to determine the corresponding applied force. Multiple updates to the material properties and failure assessments may be required during each load increment. The process of incrementally applying a displacement and altering material properties continues beyond maximum load to facilitate a more thorough understanding of the load-deflection response and failure process. 


\section{Results and Discussion}

This section is divided into two sub-sections. The first section presents typical performance results associated with single-hole specimens having different reinforcement architectures, reinforcement thicknesses and geometric ratios (W/d and e/d). A discussion of the progressive failure and final specimen failure mode of the specimens is also presented. The second section presents performance results and a discussion of the failure mechanisms associated with multiple-hole specimens.

\section{A. Single-hole specimens}

Typical load-deflection curve results for single-hole specimens with strip and circular reinforcements and nonreinforced specimens having $\mathrm{W} / \mathrm{d}=4$ and $\mathrm{e} / \mathrm{d}=4$, are presented in Fig. 4 and 5. Load-displacement results for geometrically identical non-reinforced specimens are also included. Comparison between reinforced and nonreinforced specimens is made using the same finite element mesh to eliminate any dissimilar mesh issues.

\section{Material failure modes}

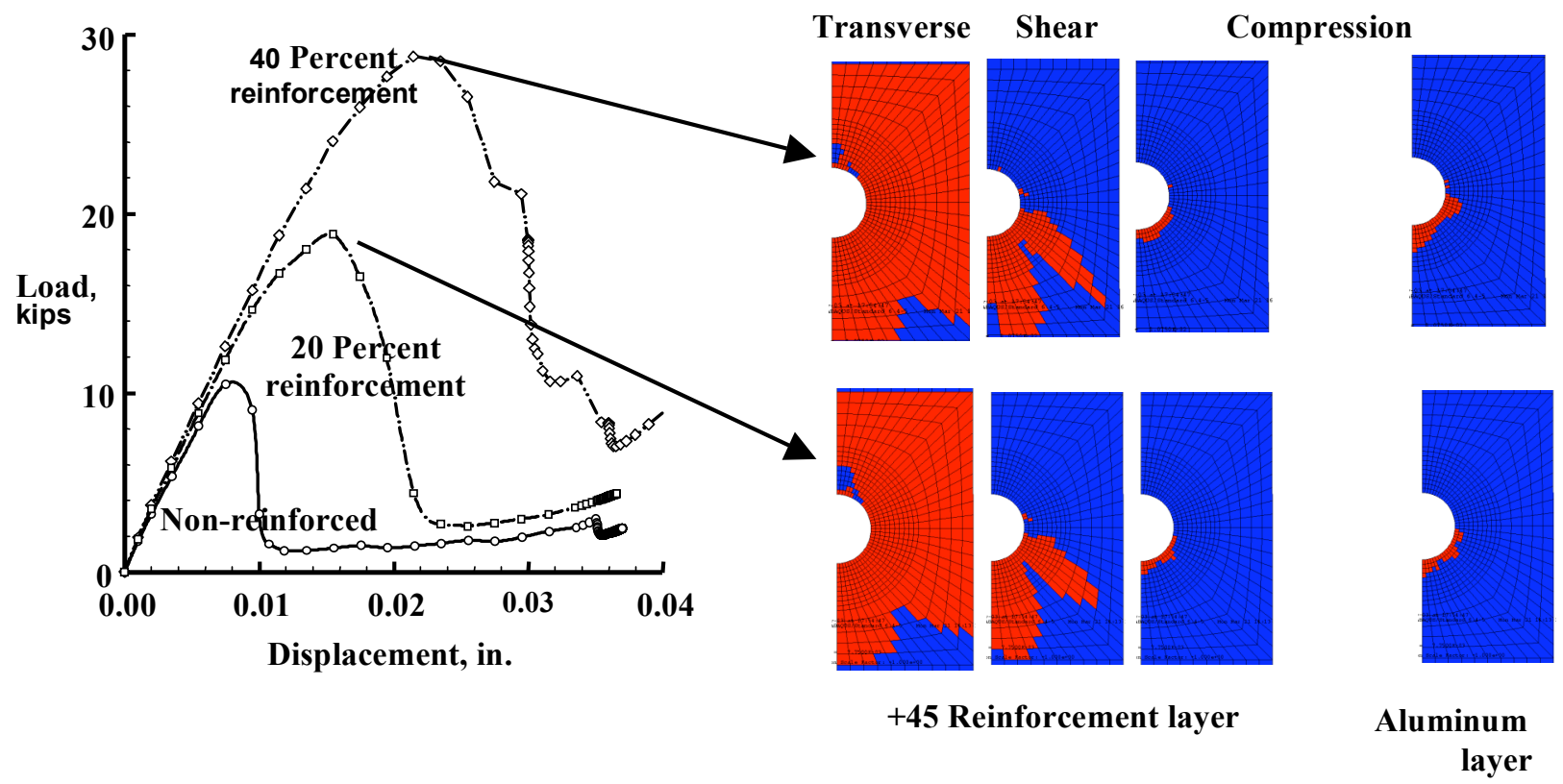

Figure 4. Load-displacement response for strip-reinforced and non-reinforced single-hole specimens $(W / d=4, e / d=4)$. 
Material failure modes

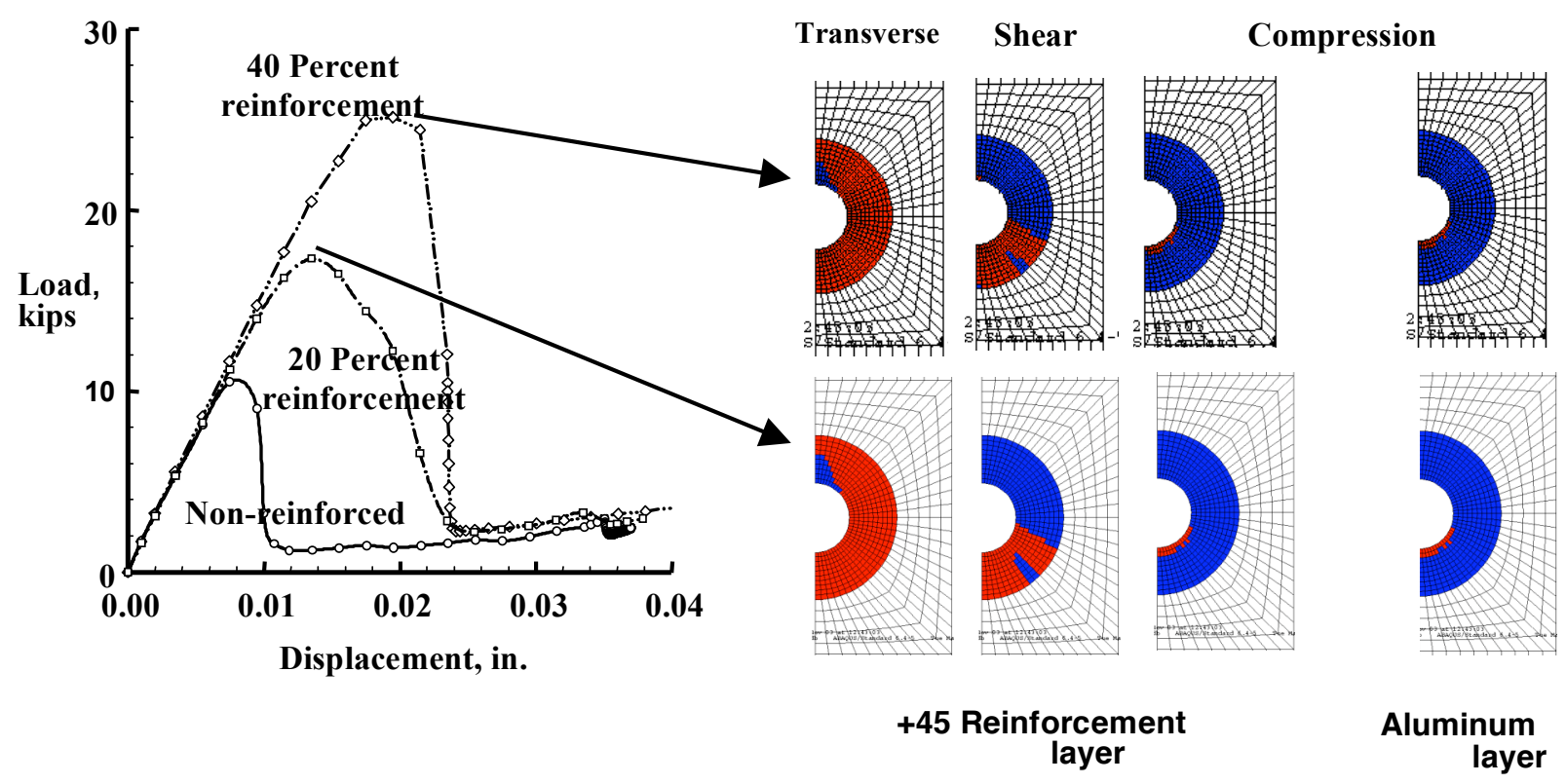

Figure 5. Load-displacement response for circular-reinforced and non-reinforced single-hole specimens $(\mathrm{W} / \mathrm{d}=4, \mathrm{e} / \mathrm{d}=4)$.

For both the 20 and 40 percent thickness reinforcement cases the maximum load of the strip reinforced specimen, in Fig. 4, was approximately 15 percent higher than the circular reinforced specimens, in Fig. 5, even though the volume of strip reinforcement was several times that of the circular reinforcement. These results demonstrate that even when locally reinforcing a region a small quantity of reinforcement strategically applied can have a significant influence on the results. The strip reinforced specimens exhibited an approximately 70 and 160 percent increase in maximum load for the 20 and 40 percent thickness reinforcement cases, respectively relative to non-reinforced specimens. Circular reinforced specimens exhibited an approximately 60 and 130 percent increase in maximum load for the 20 and 40 percent thickness reinforcement cases, respectively.

Initial failure of the reinforced specimens occurred at loads substantially below maximum load and damage progressed with continued application of end displacement. Material failure modes at maximum load are shown for both strip and circular reinforcement cases in Fig. 4 and 5, respectively.

The load-displacement curves for both reinforced architectures and non-reinforced specimens were nearly linear until near maximum load where a pronounced rounding of the response curve occurred. The material failure modes in the reinforced specimen are associated with the +45 reinforcement layer and the base aluminum beneath the reinforcement. The failure modes of the -45 reinforcement layer are similar to the +45 layer and are therefore omitted. The red (lighter) shaded region represents the damaged area whereas the blue (darker) shaded region represents undamaged portion of the reinforcement region.

Both the strip and circular reinforced specimens exhibited similar material failure patterns and failure progression even though there is considerable difference in reinforcement architectures. Both reinforcement architectures exhibited almost total transverse material failure in the reinforcement at maximum load. Shear failure occurred along the lower portion of the hole and extended out in a radial direction toward the edge of the reinforcement. Only a small region located along the lower perimeter of the hole exhibited compression failure. Although initial transverse and shear failure occurred substantially below maximum load, the nonlinearity in the load-displacement curve is related to the initiation of compression failure in the reinforcement. Material compression failure progressively grew with increase pin displacement even after maximum load was achieved. 
The base aluminum beneath the reinforcement experienced failure similar in magnitude as the compression failure in the reinforced layer.

Load is reacted in non-reinforced specimens at the edge of the hole. Material yielding occurs and the load carrying capability of the non-reinforced specimen diminishes almost immediately. However, the reinforcement in the reinforced specimens distributes the load from the edge of the hole to a broader area allowing the reinforced specimens to react higher loads than conventional non-reinforced specimens. Even though initial material failure and subsequent progression of failure of the reinforcement occurs substantially below the maximum load the loaddisplacement response is linear for $80-90$ percent of the response curve.

A limited parametric study was conducted investigating the influence of specimen $\mathrm{W} / \mathrm{d}$ and e/d ratios on percent increase in maximum load of strip reinforced single-hole specimens with results presented in Fig. 6. Specimens having 20 and 40 percent thickness reinforcements were included. All of the specimens in the W/d ratio study have an e/d ratio of 4 .

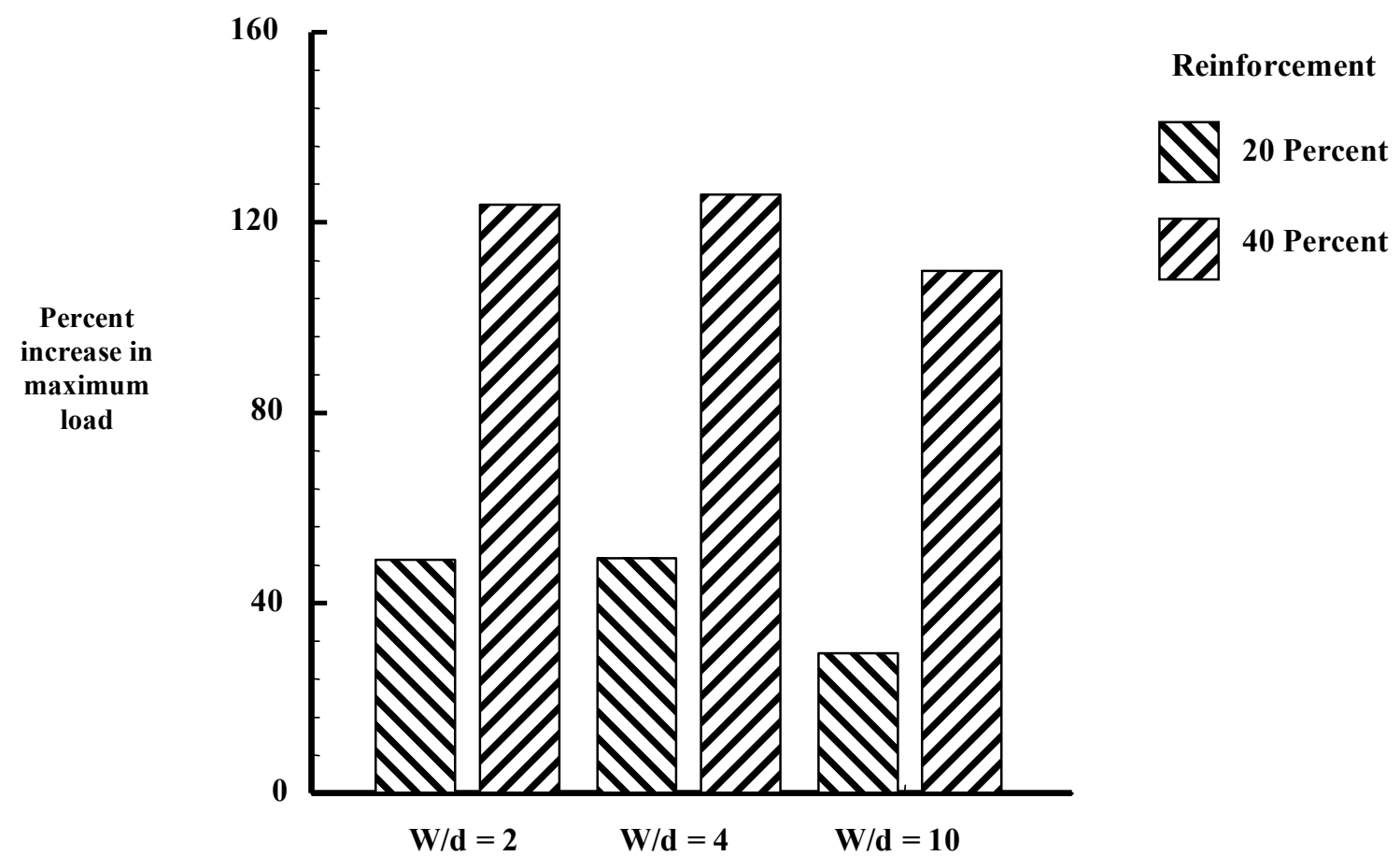

Figure 6. Percent increase in maximum load of strip-reinforced single-hole specimens as a function of $W / d$ ratio $(e / d=4)$.

For a W/d ratio of 2 the percent increase in maximum load as compared to geometrically comparable nonreinforced specimens was approximately 49 and 123 percent for 20 and 40 percent reinforcement thickness, respectively. For a value of $\mathrm{W} / \mathrm{d}$ equal to 4 , the percent increase in maximum load slightly increased for both 20 and 40 percent thick reinforced specimens relative to the $\mathrm{W} / \mathrm{d}=2$ case. For $\mathrm{W} / \mathrm{d}=10$ the percent increase in maximum load substantially decreased relative to the $\mathrm{W} / \mathrm{d}=4$ case. Maximum load nominally increases with increasing W/d ratio and in this case the maximum load of the non-reinforced specimen increased proportionally more than the corresponding reinforced specimen producing a decrease in percent increase in maximum load relative to the performance of the $\mathrm{W} / \mathrm{d}=4$ specimen. Conversely, for $\mathrm{W} / \mathrm{d}=10$ percent increase in maximum load was approximately 30 and 110 percent for the 20 and 40 percent thick reinforcement specimens, respectively. As 
specimen $\mathrm{W} / \mathrm{d}$ ratio increased there was a transition in failure mode from net-tension $(\mathrm{W} / \mathrm{d}=2)$ to a combined shearout and bearing $(\mathrm{W} / \mathrm{d}=4)$ to a bearing $(\mathrm{W} / \mathrm{d}=10)$, as depicted in Fig. 7.

$\mathbf{W} / \mathbf{d}=\mathbf{2}$

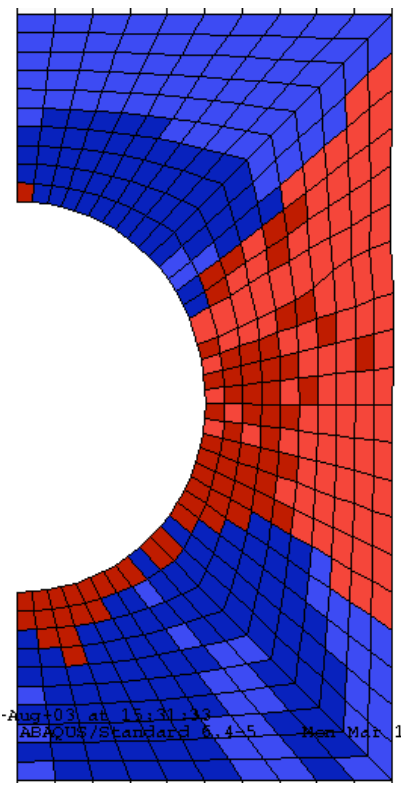

Net-tension
$\mathbf{W} / \mathbf{d}=\mathbf{4}$

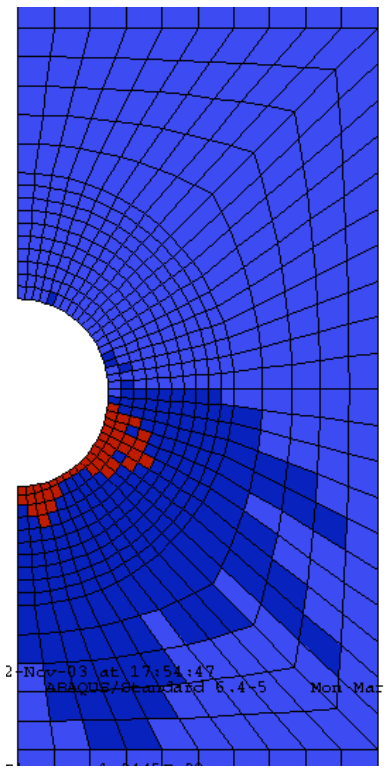

Combined bearing and shear
$\mathbf{W} / \mathbf{d}=\mathbf{1 0}$

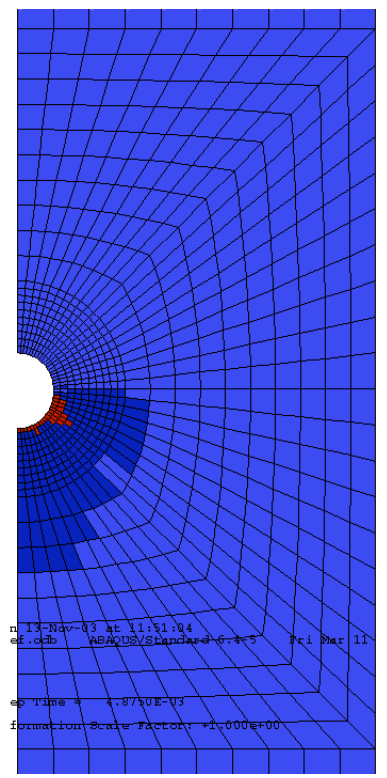

Bearing

Figure 7. Specimen failure modes represented by superposition of material shear and compression failure modes for strip-reinforced single-hole specimens $(\mathrm{e} / \mathrm{d}=4)$.

The influence of e/d ratio (distance from center of pin to end of specimen divided by hole diameter) is investigated for specimens having a W/d ratio of 4 . Reinforcement thickness of 20 and 40 percent of the total specimen thickness are included. A range of percent increase in maximum load was between 38 and 125, as depicted in Fig. 8. As e/d ratio increases from 1 to 5 there is a decrease in the percent increase in maximum load for the 20 percent thick reinforcement case. For the 40 percent thick reinforcement case percent increase in maximum load remained almost constant for e/d between 1 and 4 and substantially decreased for the e/d=5 case. For the case of e/d=1 increase in maximum load was approximately 68 and 124 percent for the 20 and 40 percent thickness reinforcement cases, respectively relative to non-reinforced specimens. Percent increase in maximum load decreased to approximately 38 and 102 percent for the 20 and 40 percent thickness reinforcement cases, respectively, for specimens having an e/d ratio of 5. This trend is logical because when the edge of the specimen is close to the center of the hole, low value of e/d, there is an adverse geometrical influence on the stress state at the bottom of the hole. Therefore, application of reinforcement in this situation would have more influence on the load carrying capability. At high values of e/d the application of reinforcement still increases the hole strength, however the influence of the specimen edge diminishes and the percent increase in maximum load due to the incorporation of reinforcement decreases. The failure mode for these specimens was a combined bearing and shear-out as depicted in Fig. 9.

\section{B. Multi-hole specimens}

Four multi-hole specimen configurations with circular reinforcement were investigated and the results presented in Fig. 10. The type of multi-hole specimens are two holes in a line, a row of holes, a combination of two rows of holes with holes in a line and two rows of holes with the holes staggered, as depicted in Fig. 2. For comparison purposes the results for a single-hole specimen are included with the results of the multi-hole specimens. As was the case for the single-hole specimens two reinforcement thicknesses, 20 and 40 percent, are included in this investigation. 


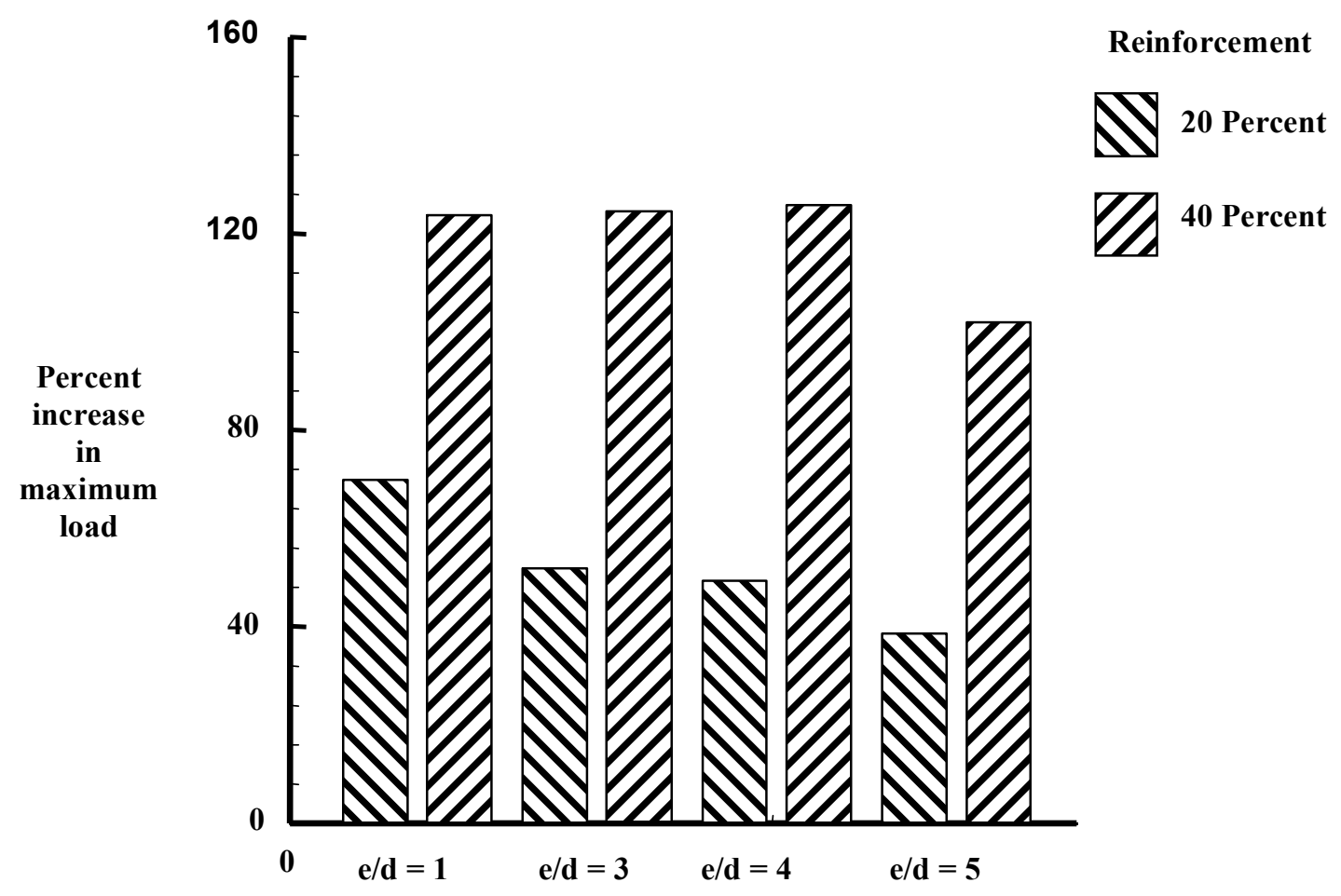

Figure 8. Percent increase in maximum load of strip-reinforced single-hole specimens as a function of e/d ratio $(W / d=4)$. 


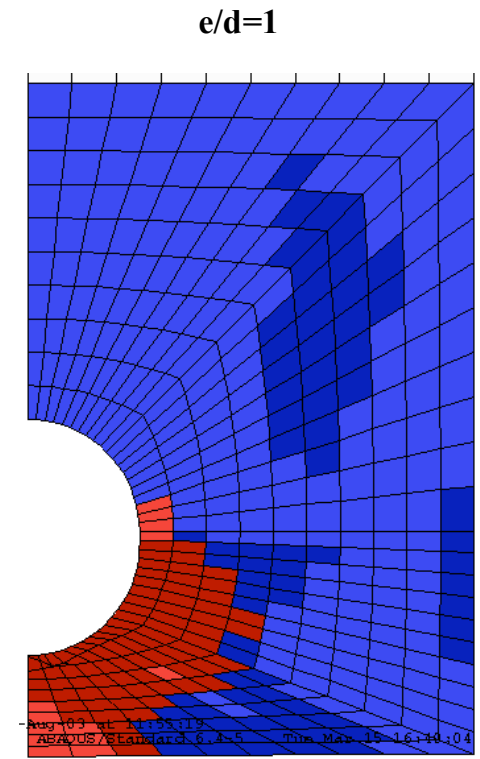

Combined bearing and shear $\mathbf{e} / \mathbf{d}=\mathbf{3}$

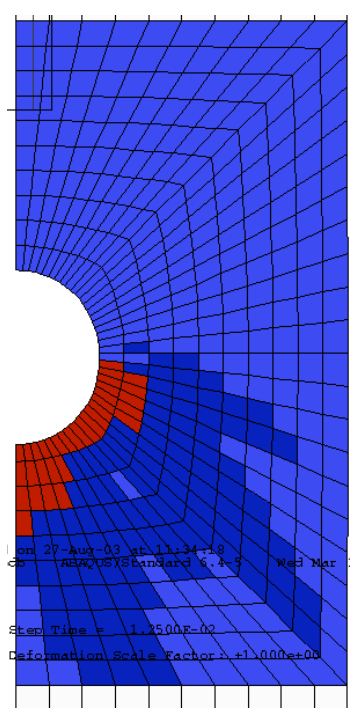

Combined bearing and shear $\mathrm{e} / \mathrm{d}=\mathbf{5}$

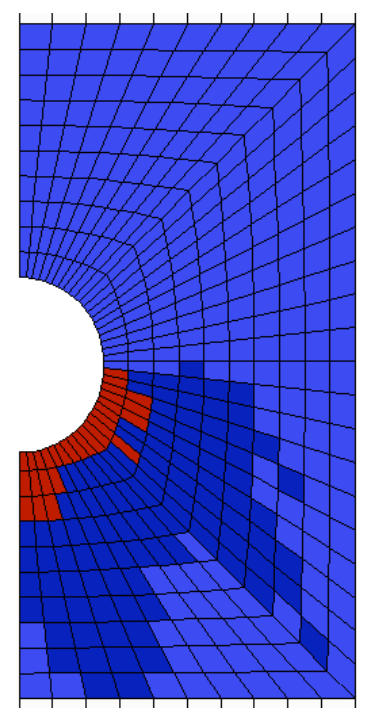

Combined bearing and shear

Figure 9. Specimen failure modes represented by superposition of material shear and compression failure modes for strip-reinforced single-hole specimens $(W / d=4)$.

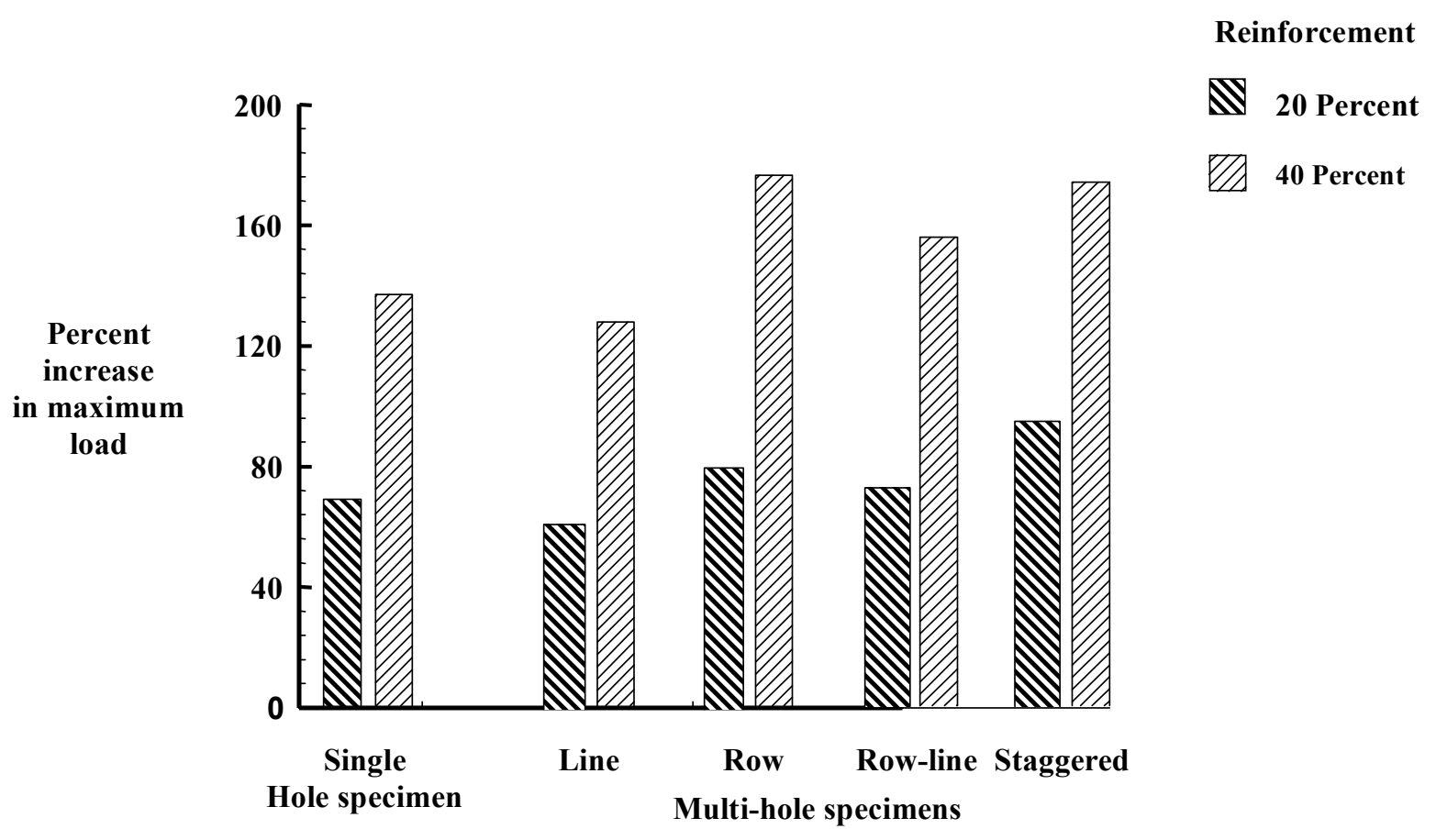

Figure 10. Percent increase in maximum load for multiple-hole specimens with circular reinforcements $(\mathrm{W} / \mathrm{d}=4, \mathrm{e} / \mathrm{d}=4)$. 
In all cases the percent increase in maximum load for reinforced multi-hole specimens resulted in a significant improvement, 60 to 175 percent, relative to the performance of non-reinforced specimens. The range of improvement was consistent with the results obtained for the single-hole specimen. In the case of the line of bolts the percentage improvement for the reinforced specimen was less than that achieved for the single-hole specimen. Percent increase in maximum load did not scale proportionally with reinforcement thickness for all multi-hole specimens but in general the results were close.

A detailed discussion of the load-deflection response and progressive failure of the staggered-hole specimen is presented. A similar discussion is not presented for the other multiple-hole specimens because of their similar responses. The load-deflection response of the staggered multi-hole specimen for 40 percent thick and nonreinforced case is presented in Fig. 11. Images of the Mises stress state at maximum load in the aluminum are included in Fig. 11. Material failure modes in the reinforcement and in the base aluminum beneath the reinforcement are depicted in Fig. 12.

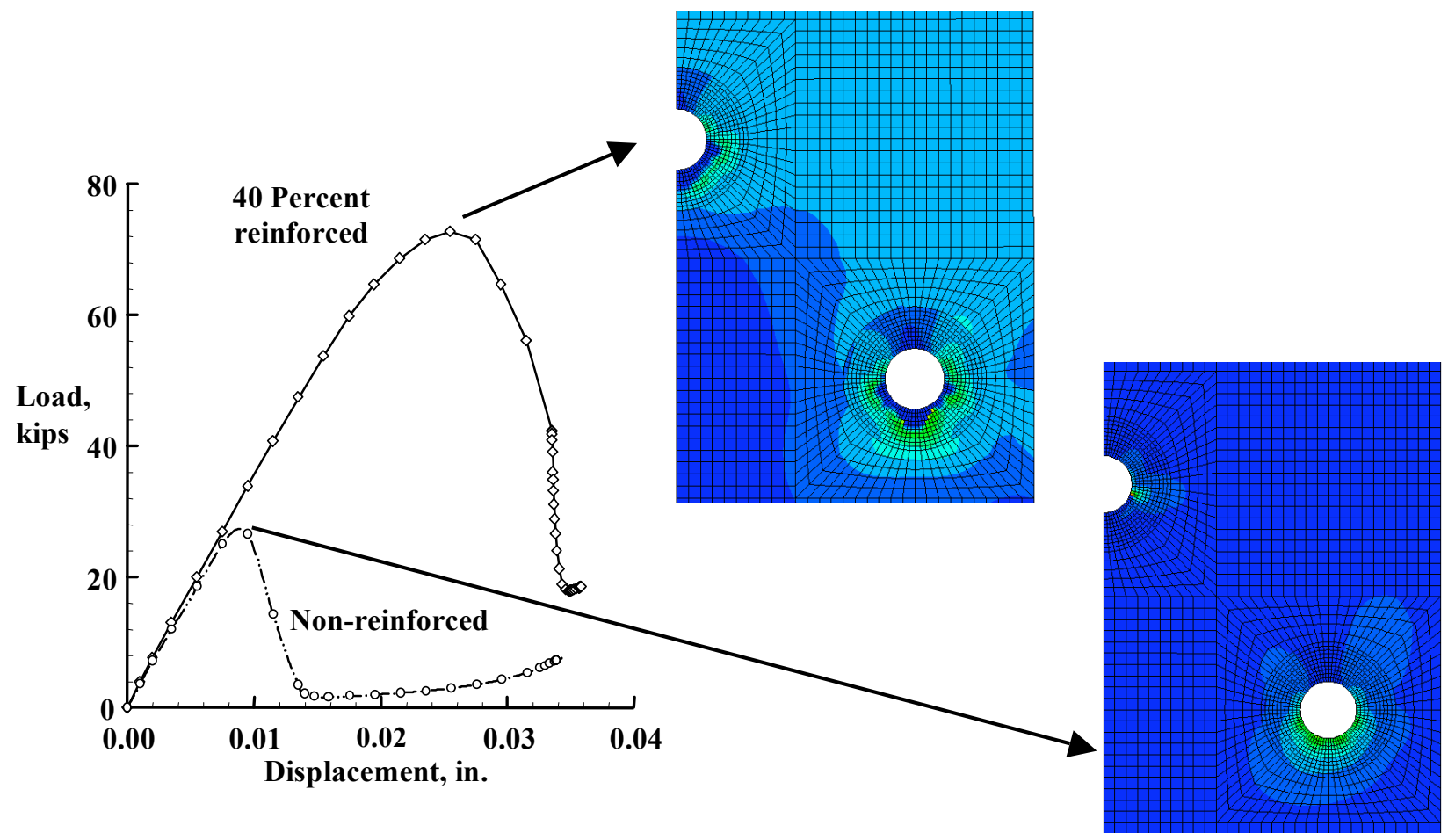

Figure 11. Load-displacement response of 40 percent thick and non-reinforced staggered hole specimens and Mises stresses at maximum load in aluminum $(W / d=4)$.

A parabolic shaped load-deflection curve was predicted for the staggered multi-hole specimen, which is similar to that of the reinforced single-hole specimen. The circular reinforcement seems to create a better distribution of load in the loaded-hole region than is otherwise produced without reinforcement. The non-reinforced specimen exhibits a more concentrated stress state around the hole. Even when yielding in the aluminum beneath the reinforcement occurs the reinforcement is able to redistribute the load from the yielded aluminum over a broader area resulting in the ability to react higher loads.

Initial material failure in the reinforcement was predicted well below the maximum load experienced by the specimen similar to that exhibited by the single-hole specimens. Initial material failure was transverse tension. As load increased a shear material failure mechanism occurred and propagated in the reinforcement. Yielding in the base aluminum and compression failure in the reinforcement initiated at approximately 85 percent of the maximum 
load. Load-deflection response was linear until approximately 85 percent of maximum load was achieved. Until this point the progression of failure in the reinforcement had little or no apparent influence on the response. Reinforcement fibers in compression were the primary load carrying mechanism in the reinforcement material at and beyond the 85 percent of the maximum load state. The parabolic nature of the load-deflection response, during unloading, continued to approximately 60 percent of maximum load. Beyond this point the slope of the loaddeflection response became nearly vertical and a significant increase in compression failure in the reinforcement occurred. The final material failure did not propagate in the base aluminum beyond the reinforcement region.

Transverse

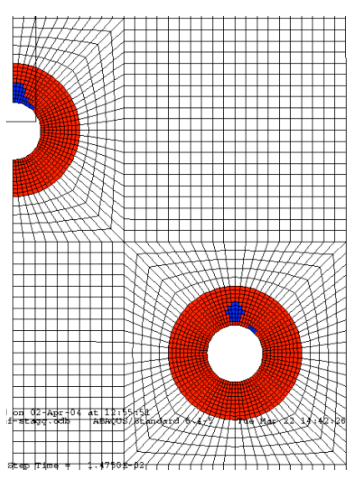

Shear

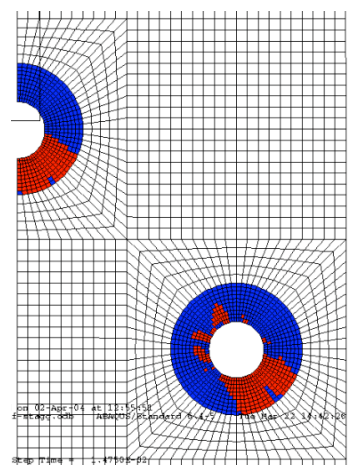

+45 Reinforcement

layer

\section{Compression}
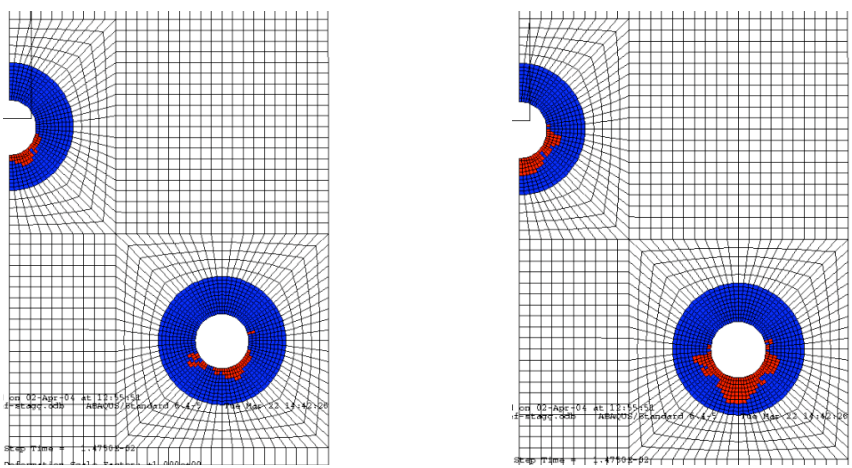

Aluminum layer

Figure 12 - Material failure modes for 40 percent thick staggered multiple-hole specimen at maximum load.

\section{Conclusion}

An analysis based investigation of selectively reinforced rigid-pin loaded single- and multi-hole specimens was performed and the results are compared to those of geometrically comparable specimens without reinforcements. Reinforcement thicknesses of 20 and 40 percent of the total specimen thickness were used in this investigation. Two reinforcement architectures, strip and circular, were also employed. Both reinforcement architectures had fiber orientations of \pm 45 degrees. For all cases investigated selective reinforcement has a significant positive influence on the response of single- and multi-hole specimens. The following concluding remarks seem warranted.

1. Percentage increase in maximum load ranged from 30 to over 170 without increasing the total thickness of the specimen. Selective reinforcement distributes load away from the edge of the hole producing higher maximum load.

2. The failure modes exhibited by the selectively reinforced specimens (net-tension, shear-out and bearing) were consistent with failure modes exhibited by fiber reinforced polymer matrix composites specimens.

3. Circular and strip reinforcement architectures are both effective reinforcement solutions. Specimens with circular reinforcements had lower percentage increase in maximum load than the strip reinforced specimens. However, the quantity of reinforcement material used for circular reinforcement was substantially less than the strip reinforcement.

4. Increase in maximum load for selectively reinforced specimens does not always scale proportionally with total reinforcement thickness. Non-scaling of results is attributed to small changes in local material failure.

\section{References}

${ }^{1}$ Garratt, M. D., Bucci, R. J., and Kulak, M., “FCG Evaluation of New Lower Wing Concept,” AeroMat Conference, June 7-10, 2004. 
${ }^{2}$ Farley, Gary L., "Selective Reinforcement To Enhance The Structural Performance of Metallic Compression Panels," $45^{\text {th }}$ AIAA/ASME/ASCE/AHS/ASC/SDM Conference, Palm Springs, CA, April 19-22, 2004.

${ }^{3}$ Farley, Gary L., Newman, John A., and James, Mark A., "Selective Reinforcement To Improve Fracture Toughness and Fatigue Crack Growth Resistance In Metallic Structures," 45 ${ }^{\text {th }}$ AIAA/ASME/ASCE/AHS/ASC/SDM Conference, Palm Springs, CA, April 19-22, 2004.

${ }^{4}$ Padawer, G. E., “Film Reinforced Multi-Fastened Mechanical Joints In Fibrous Composites”, AIAA Paper No. 72-382, 1972.

${ }^{5}$ Anon., ABAQUS users manual, Hibbitt, Karlsson and Sorensen, Inc., 2000.

${ }^{6}$ Hashin, Z., " Failure Criteria for Unidirectional Fiber Composites,", Journal of Applied Mechanics, June 1980, Vol. 47, pp. 329334. 